PHYSICAL REVIEW D 96, 069901(E) (2017)

\title{
Erratum: Higgs portal to inflation and fermionic dark matter [Phys. Rev. D 93, 123513 (2016)]
}

Aditya Aravind, Minglei Xiao, and Jiang-Hao Yu

(Received 25 August 2017; published 26 September 2017)

\begin{abstract}
The beta functions for various coupling coefficients in the Lagrangian (Appendix A in the paper) have been corrected and the updated version of the plots in the paper have been included. The conclusions of the paper remain the same.
\end{abstract}

DOI: 10.1103/PhysRevD.96.069901

\section{CORRECTED BETA FUNCTIONS}

The following is the corrected version of the beta functions [Eqs. (31)] reported in Appendix A of the paper. The beta functions for $\lambda_{s h}, \lambda_{s}, y_{\psi}, \xi_{h}$, and $\xi_{s}$ have been corrected. Additionally, the notations $\xi_{h}$ and $\xi_{\phi}$ were interchangeably used in these equations and have now been consistently referred to as $\xi_{h}$ to match with the rest of the paper. Also, a typographical error has been corrected in the beta function for $y_{t}$ and additional terms have been included for better accuracy.

$$
\begin{aligned}
\beta_{g_{s}}= & \frac{g_{s}^{3}}{(4 \pi)^{2}}(-7)+\frac{g_{s}^{3}}{(4 \pi)^{4}}\left(\frac{11}{6} g^{\prime 2}+\frac{9}{2} g^{2}-26 g_{s}^{2}-2 x_{\phi} y_{t}^{2}\right), \\
\beta_{g}= & \frac{g^{3}}{(4 \pi)^{2}}\left(-\frac{39-x_{\phi}}{12}\right)+\frac{g^{3}}{(4 \pi)^{4}}\left(\frac{3}{2} g^{\prime 2}+\frac{35}{6} g^{2}+12 g_{s}^{2}-\frac{3}{2} x_{\phi} y_{t}^{2}\right), \\
\beta_{g^{\prime}}= & \frac{g^{\prime 3}}{(4 \pi)^{2}}\left(\frac{81+x_{\phi}}{12}\right)+\frac{g^{\prime 3}}{(4 \pi)^{4}}\left(\frac{199}{18} g^{\prime 2}+\frac{9}{2} g^{2}+\frac{44}{3} g_{s}^{2}-\frac{17}{6} x_{\phi} y_{t}^{2}\right), \\
\beta_{\lambda_{h}}= & \frac{1}{(4 \pi)^{2}}\left(6\left(1+3 x_{\phi}^{2}\right) \lambda_{h}^{2}-6 y_{t}^{4}+\frac{3}{8}\left(2 g^{4}+\left(g^{2}+g^{\prime 2}\right)^{2}\right)+\lambda_{h}\left(-9 g^{2}-3 g^{\prime 2}+12 y_{t}^{2}\right)+\frac{1}{2} \lambda_{s h}^{2} x_{s}^{2}\right), \\
\beta_{\lambda_{s h}=} & \frac{\lambda_{s h}}{(4 \pi)^{2}}\left(6\left(x_{\phi}^{2}+1\right) \lambda_{h}+4 x_{\phi} x_{s} \lambda_{s h}+6 x_{s}^{2} \lambda_{s}+6 y_{t}^{2}+4 y_{\psi}^{2}-\frac{9}{2} g^{2}-\frac{3}{2} g^{\prime 2}\right), \\
\beta_{\lambda_{s}}= & \frac{1}{(4 \pi)^{2}}\left(18 x_{s}^{2} \lambda_{s}^{2}+8 \lambda_{s} y_{\psi}^{2}+\frac{1}{2}\left(x_{\phi}^{2}+3\right) \lambda_{s h}^{2}-8 y_{\psi}^{4}\right), \\
\beta_{y_{\psi \prime}}= & \frac{5 x_{s} y_{\psi}^{3}}{(4 \pi)^{2}}, \\
\beta_{y_{t}}= & \frac{y_{t}}{(4 \pi)^{2}}\left[-\frac{9}{4} g^{2}-\frac{17}{12} g^{\prime 2}-8 g_{s}^{2}+\frac{23+4 x_{\phi}}{6} y_{t}^{2}\right] \\
& +\frac{y_{t}}{(4 \pi)^{4}}\left[-\frac{23}{4} g^{4}-\frac{3}{4} g^{2} g^{\prime 2}+\frac{1187}{216} g^{\prime 4}+9 g^{2} g_{s}^{2}+\frac{19}{9} g^{\prime 2} g_{s}^{2}-108 g_{s}^{4}\right. \\
& \left.+\left(\frac{225}{16} g^{2}+\frac{131}{16} g^{\prime 2}+36 g_{s}^{2}\right) x_{\phi} y_{t}^{2}+6\left(-2 x_{\phi}^{2} y_{t}^{4}-2 x_{\phi}^{3} y_{t}^{2} \lambda_{h}+x_{\phi}^{2} \lambda_{h}^{2}\right)\right], \\
\beta_{\xi_{h}}= & \frac{1}{(4 \pi)^{2}}\left[\left(\xi_{h}+\frac{1}{6}\right)\left[-\frac{3}{2} g^{\prime 2}-\frac{9}{2} g^{2}+6 y_{t}^{2}+\left(6+6 x_{\phi}\right) \lambda_{h}\right]+\left(\xi_{s}+\frac{1}{6}\right) x_{s} \lambda_{s h}\right], \\
\beta_{\xi_{s}}= & \frac{1}{(4 \pi)^{2}}\left[\left(\xi_{s}+\frac{1}{6}\right)\left(6 x_{s} \lambda_{s}\right)+\left(\xi_{h}+\frac{1}{6}\right)\left(x_{\phi}+3\right) \lambda_{s h}\right] .
\end{aligned}
$$

Here, $g_{s}, g, g^{\prime}$, and $y_{t}$ are the standard model $S U(3), S U(2), U(1)$ and top-quark Yukawa couplings, $\lambda_{h}$ and $\lambda_{s}$ are the quartic self-couplings of Higgs and $s$-fields, $\lambda_{s h}$ is the quartic cross-coupling, $\xi_{h}$ and $\xi_{s}$ are the nonminimal couplings of the two fields to gravity, and additionally we also define 


$$
x_{\phi}=\frac{1+\xi_{h} h^{2} / M_{\mathrm{pl}}^{2}}{1+\xi_{h} h^{2} / M_{\mathrm{pl}}^{2}+6 \xi_{h}^{2} h^{2} / M_{\mathrm{pl}}^{2}}, \quad x_{s}=\frac{1+\xi_{s} s^{2} / M_{\mathrm{pl}}^{2}}{1+\xi_{s} s^{2} / M_{\mathrm{pl}}^{2}+6 \xi_{s}^{2} s^{2} / M_{\mathrm{pl}}^{2}} .
$$

\section{IMPLICATIONS}

In the paper, the beta functions are used to obtain the running of the couplings from electroweak scale to the inflationary scale, using which the RG-corrected potential of the inflaton field is computed. Therefore, changing the beta functions can potentially change the shape of the inflationary potential and thereby affect the inflationary $\left(n_{s}-r\right)$ predictions. Our updated plots indicate that the changes do not drastically or qualitatively affect the results of the paper. This is expected, because the original loop corrections also did not qualitatively modify the prediction coming from the tree-level Higgs inflation other than allowing for a range of $n_{s}-r$ values around the tree-level value.

Our methodology in making the correction is as follows: We started with the existing data points which still agree with the dark matter and collider constraints which are unmodified by the beta functions since they pertain to electroweak scale physics. We then used the corrected beta functions to compute the running values of these couplings which were then used to calculate the inflationary potential and related parameters. In the process, it was observed that a small fraction of the data points (about 10\%-15\%) that originally seemed to work (with the incorrect beta functions) actually lead to unstable potentials or nonperturbative values of couplings, and therefore have to be discarded. For the remaining successful data points, the inflationary observables were calculated and the plots were updated accordingly.

\section{UPDATED PLOTS AND CONCLUSION}

All the plots in the original paper have changed. The four plots each in Fig. 1 and Fig. 2 showed the running behavior of various coupling coefficients as well as the inflationary potential. These plots naturally depend on the beta functions and have been updated.
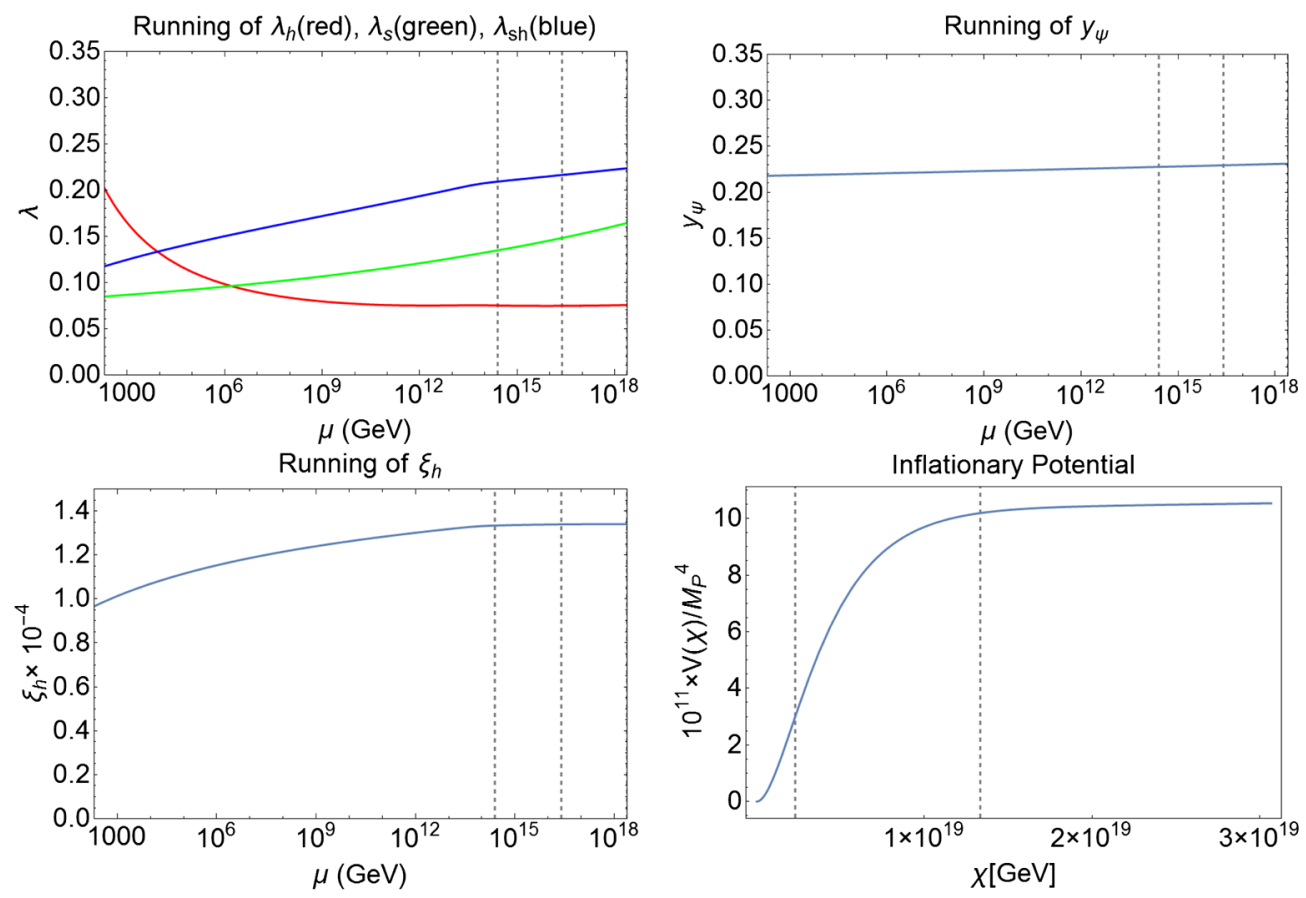

FIG. 1. Running behavior and shape of potential for $h$-inflation for (approximate) parameter values $\left\{m_{s}, m_{\psi}, u\right\}=$ $\{450,235,1081\} \mathrm{GeV}$ and $\left\{\lambda_{h}, \lambda_{s}, \lambda_{s h}, \varphi\right\}=\{0.17,0.08,0.12,0.17\}$. The plot on the top left shows the running of $\lambda_{h}, \lambda_{s}$, and $\lambda_{s h}$. The plot on the top right shows the running of $y_{\psi}$. The bottom left plot shows the running of nonminimal coupling $\xi_{h}$, and the bottom right plot shows the inflationary potential. In the first three plots, the vertical dashed lines correspond to $M_{\mathrm{pl}} / \xi_{h}$ (left) and $M_{\mathrm{pl}} / \sqrt{\xi_{h}}$ (right). In the fourth plot, they correspond to the scales of end of inflation (left) and horizon exit (right). 

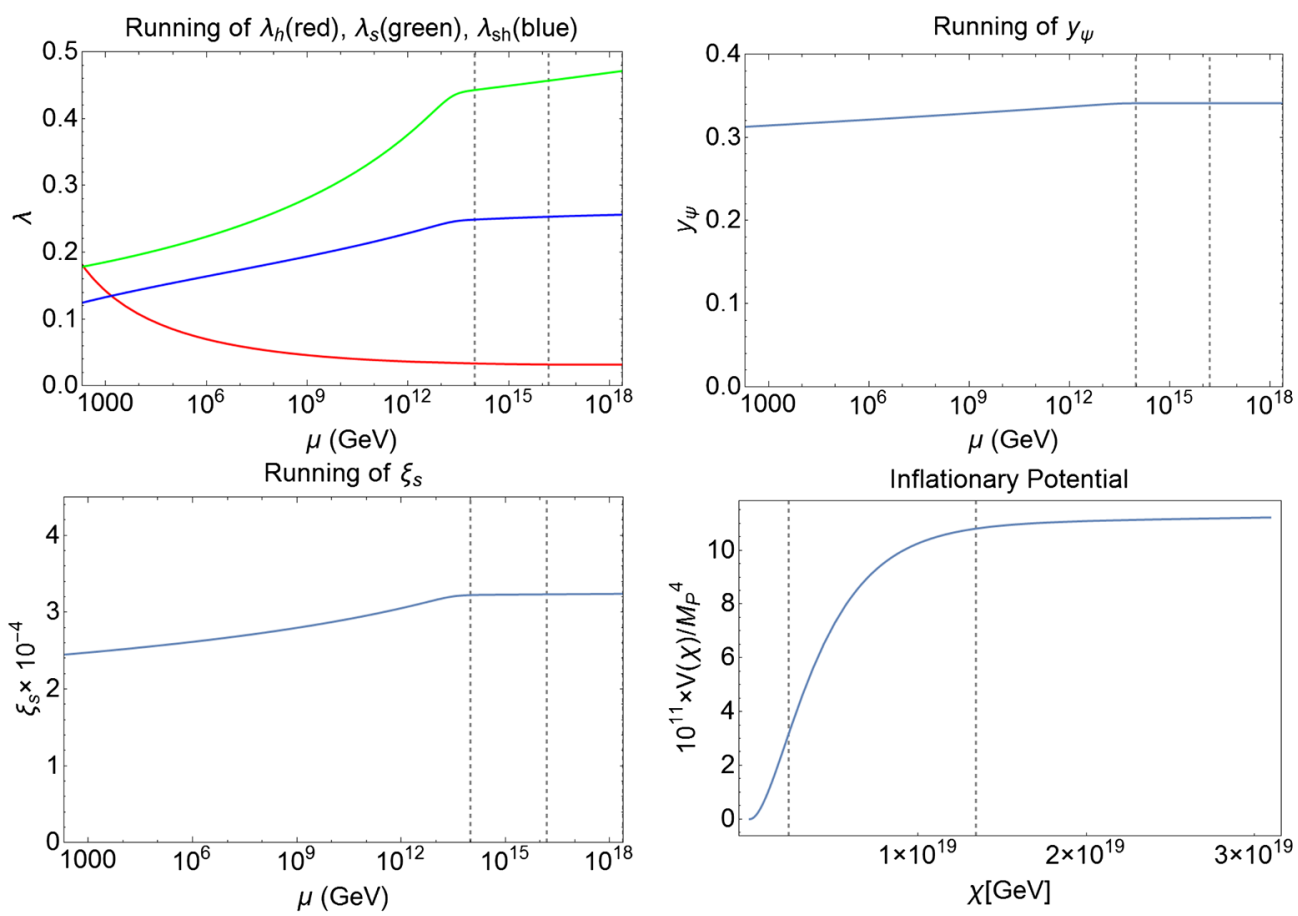

FIG. 2. Running behavior and shape of potential for $s$-inflation for (approximate) parameter values $\left\{m_{s}, m_{\psi}, u\right\}=$ $\{493,257,823\} \mathrm{GeV}$ and $\left\{\lambda_{h}, \lambda_{s}, \lambda_{s h}, \varphi\right\}=\{0.15,0.18,0.12,0.11\}$. The plot on the top left shows the running of $\lambda_{h}, \lambda_{s}$, and $\lambda_{s h}$. The plot on the top right shows the running of $y_{\psi}$. The bottom left plot shows the running of nonminimal coupling $\xi_{s}$, and the bottom right plot shows the inflationary potential. In the first three plots, the vertical dashed lines correspond to $M_{\mathrm{pl}} / \xi_{s}$ (left) and $M_{\mathrm{pl}} / \sqrt{\xi_{s}}$ (right). In the fourth plot, they correspond to the scales of end of inflation (left) and horizon exit (right).

Figures 3, 4, and 5 (2 plots) represent data corresponding to the electroweak values of various parameters. For these plots, the only change is that a few of the points have now been discarded (since they no longer give successful inflation).

The same is true with the two plots on the left in Fig. 6, which show electroweak scale values of parameters. However, the remaining two plots in Fig. 6 also include inflation-scale values parameters and therefore have been modified.

The final result of the paper $\left(n_{s}-r\right.$ predictions) presented in Fig. 7 also depends on the beta functions. There are no longer any points with $n_{s}-r$ values outside the region favored by Planck. The earlier version of the paper had a few points with very low values of $n_{s}$ which is not the case any more. Apart from this change, the conclusions of the original paper are completely unaffected.

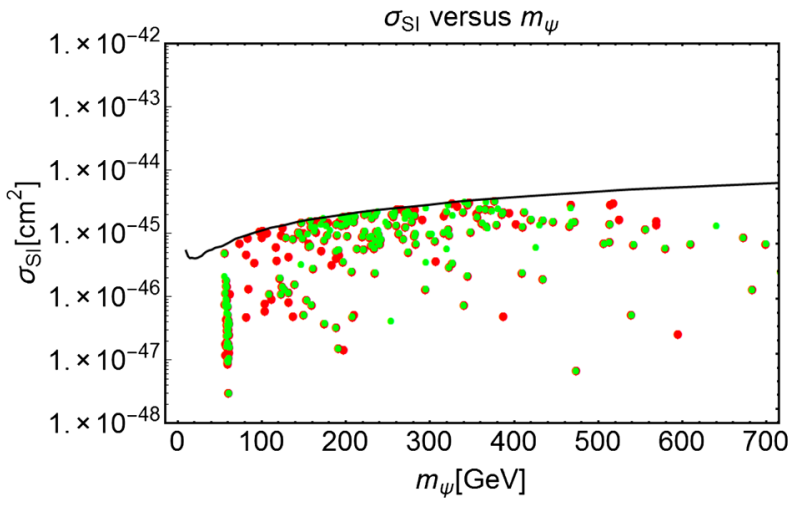

FIG. 3. Spin independent direct detection cross section $\sigma_{S I}$ plotted as a function of dark matter mass. The black line corresponds the LUX bound [1]. The green and red points correspond to $h$-inflation and $s$-inflation, respectively. 
$\varphi$ versus $m_{s}$

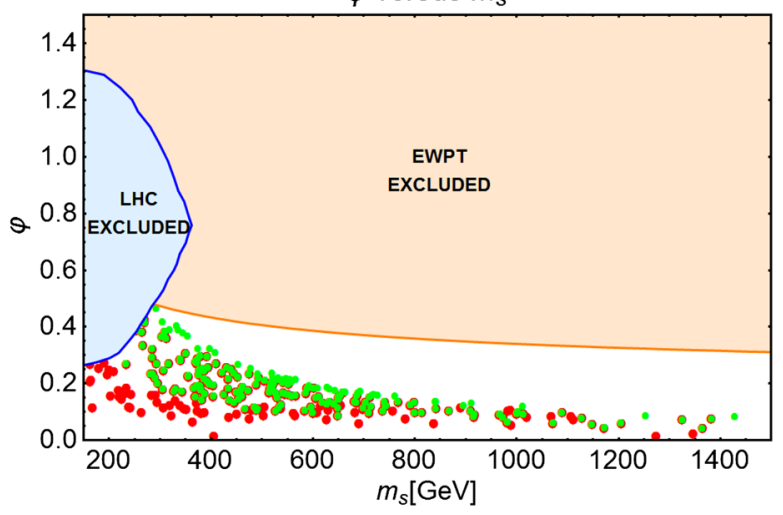

FIG. 4. Comparison of mixing angle $\varphi$ as a function of mass of the scalar field at its low energy vacuum, $m_{s}$. The orange line corresponds to the EWPT upper bound [2] and the blue line corresponds to LHC physics lower bound [3] on $m_{s}$. The orange and blue shaded regions are excluded by these bounds respectively. The green points correspond to $h$-inflation $\left(\lambda_{h}\right)$ and the red points correspond to $s$-inflation $\left(\lambda_{s}\right)$.
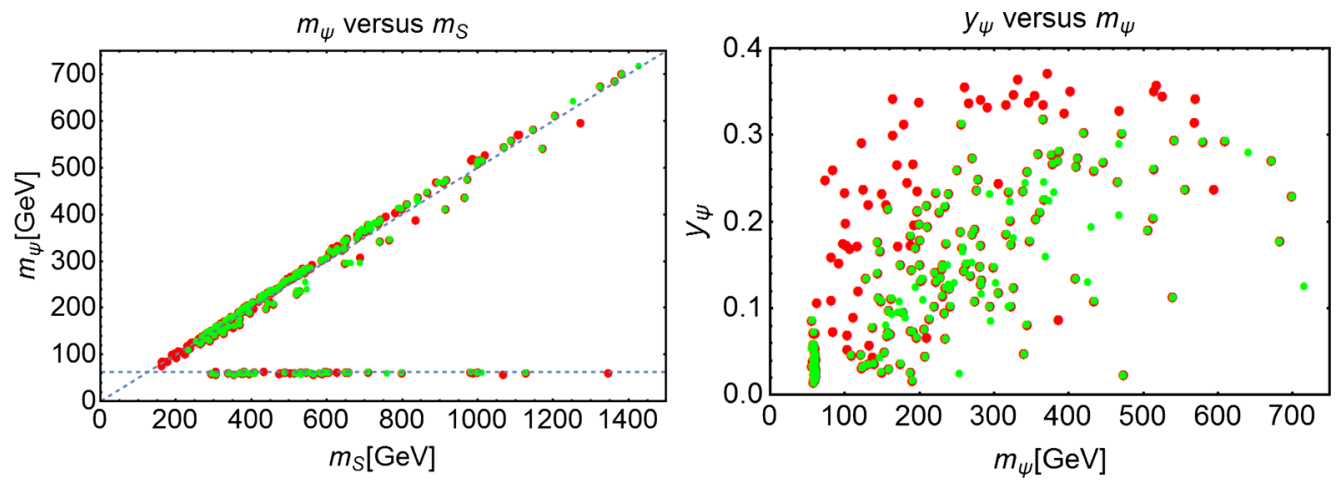

FIG. 5. On the left side, dark matter mass $m_{\psi}$ plotted against the scalar mass $m_{s}$. The dashed lines correspond to $m_{\psi}=(1 / 2) m_{s}$ and $m_{\psi}=(1 / 2) m_{h}$, respectively. On the right side, the dark matter Yukawa coupling $y_{\psi}$ is plotted against dark matter mass $m_{\psi}$. The green points correspond to $h$-inflation and the red points correspond to $s$-inflation. Note that many green points coincide with red points, indicating a potential that can support both $h$-inflation and $s$-inflation. 

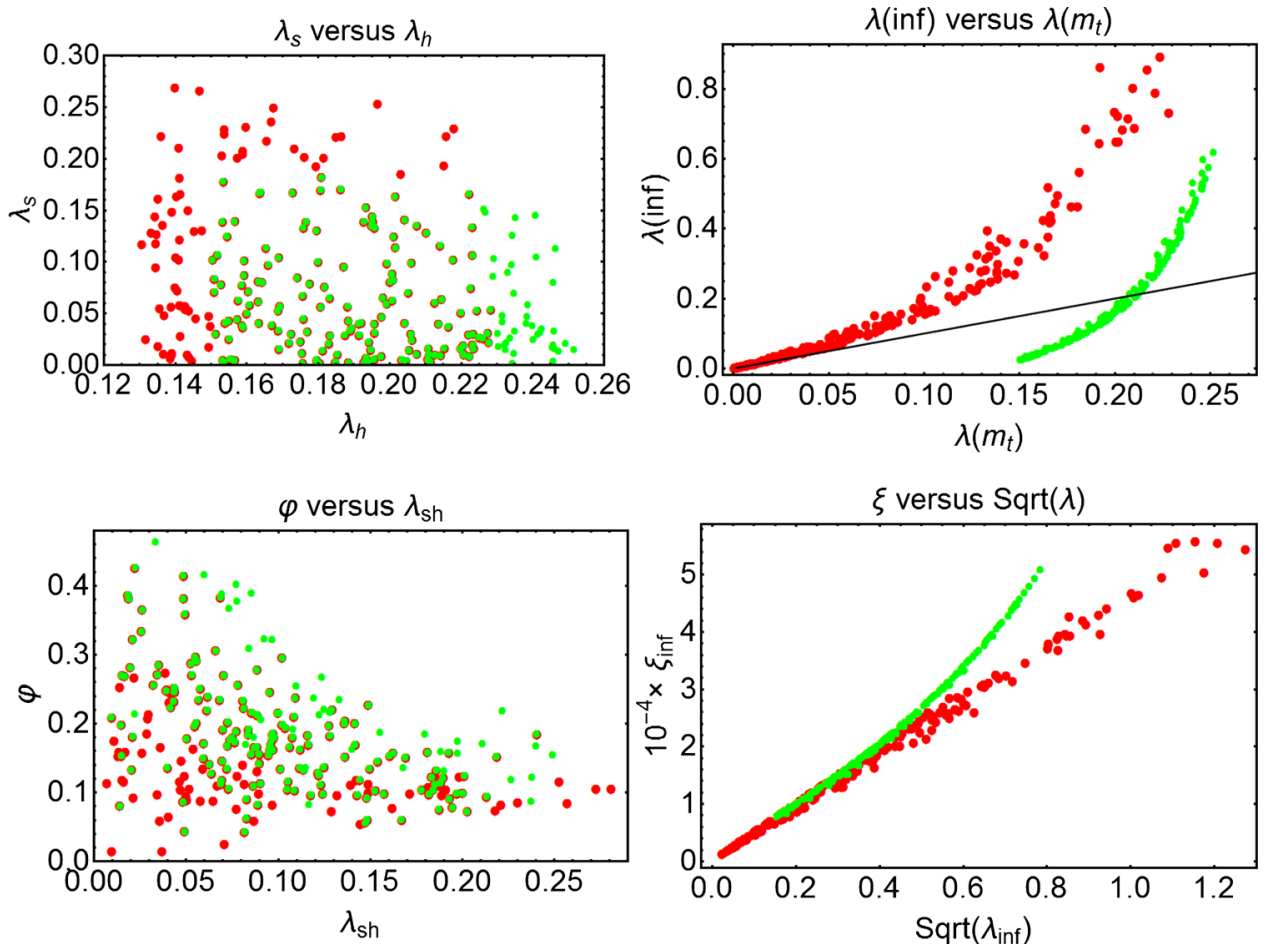

FIG. 6. The figure on the top left shows $\lambda_{h}$ and $\lambda_{s}$ at the electroweak scale. The figure on top right shows the $\lambda$ at the inflationary scale as compared to $\lambda$ at the electroweak scale, $\lambda$ being $\lambda_{h}$ or $\lambda_{s}$ for $h$ - or $s$-inflation (the black line corresponds to $y=x$ ). The figure on the bottom left shows mixing angle $\varphi$ versus $\lambda_{s h}$ at the electroweak scale. The figure on the bottom right shows $\sqrt{\lambda}$ as a function of nonminimal coupling $\xi$ evaluated at the scale of inflation. In all the plots, the green points correspond to $h$-inflation and the red points correspond to $s$-inflation.
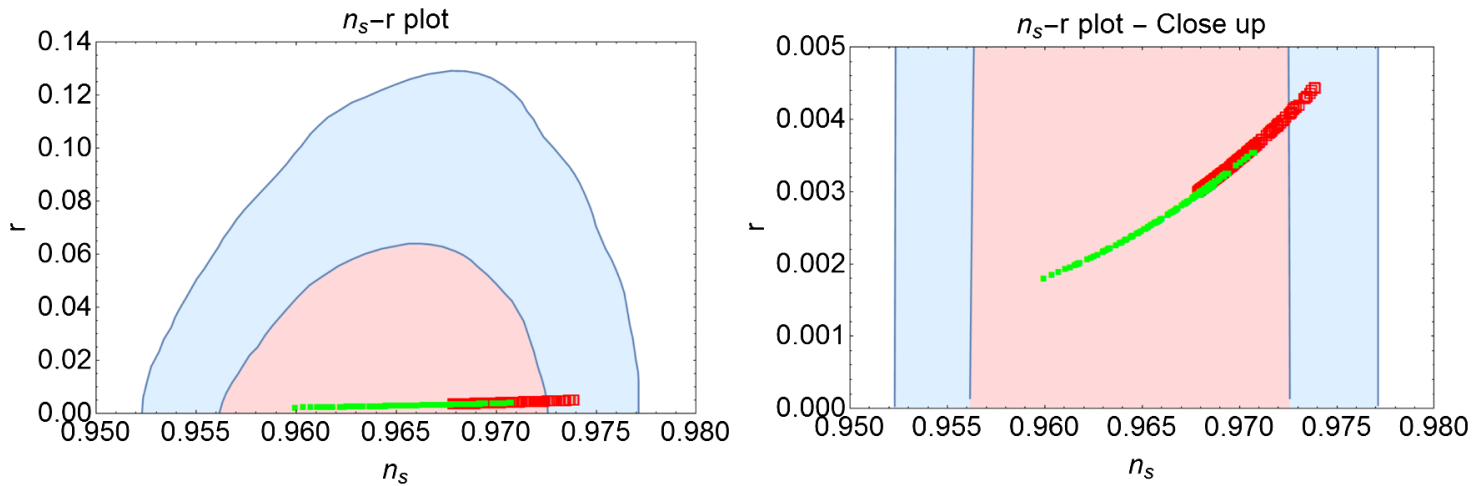

FIG. 7. $n_{s}-r$ values for $h$-inflation and $s$-inflation. The plot on the left shows the complete range of Planck 2015 [4] $68 \%$ (red) and $95 \%$ (blue) confidence limits, while the right plot zooms into the location of our data points. The filled green points (squares) correspond to $h$-inflation and the empty red points correspond to $s$-inflation. 


\section{ACKNOWLEDGMENTS}

We would like to thank Jinsu Kim for helpful discussions.

[1] D. S. Akerib et al. (LUX Collaboration), Phys. Rev. Lett. 116, 161301 (2016).

[2] S. Baek, P. Ko, W. I. Park, and E. Senaha, J. High Energy Phys. 11 (2012) 116.

[3] M. L. Xiao and J. H. Yu, Phys. Rev. D 90, 014007 (2014); 90, 019901(E) (2014).

[4] P. A. R. Ade et al. (Planck Collaboration), Astron. Astrophys. 594, A20 (2016). 